\title{
Genetic heterogeneity of porcine enteric caliciviruses identified from diarrhoeic piglets
}

\author{
V. Martella $\cdot$ K. Bányai $\cdot$ E. Lorusso $\cdot$ A. L. Bellacicco $\cdot$ N. Decaro $\cdot$ \\ V. Mari · L. Saif · V. Costantini · S. De Grazia · G. Pezzotti · A. Lavazza • \\ C. Buonavoglia
}

Received: 13 September 2007/ Accepted: 4 January 2008/Published online: 19 January 2008

(C) Springer Science+Business Media, LLC 2008

\begin{abstract}
Enteric caliciviruses (noroviruses and sapoviruses) are responsible for the majority of non-bacterial gastroenteritis in humans of all age groups. Analysis of the polymerase and capsid genes has provided evidence for a huge genetic diversity, but the understanding of their ecology is limited. In this study, we investigated the presence of porcine enteric caliciviruses in the faeces of piglets with diarrhoea. A total of 209 samples from 118 herds were analyszd and calicivirus RNA was detected by RT-PCR in 68 sample $(32.5 \%)$ and in 46 herds $(38.9 \%)$, alone or in mixed infection with group $\mathrm{A}$ and $\mathrm{C}$ rotaviruses. Sequence
\end{abstract}

V. Martella $(\bowtie) \cdot$ E. Lorusso - A. L. Bellacicco · N. Decaro V. Mari · C. Buonavoglia

Department of Animal Health and Wellbeing, Faculty

of Veterinary Medicine of Bari, S.p. per Casamassima Km 3,

70010 Valenzano, Bari, Italy

e-mail: v.martella@veterinaria.uniba.it

K. Bányai

Regional Laboratory of Virology, Baranya County Institute of State Public Health Service, Pecs, Hungary

\section{Saif · V. Costantini}

Food Animal Health Research Program, Department of Veterinary Preventive Medicine, Ohio Agricultural Research and Development Center, The Ohio State University, Wooster, OH 44691-4096, USA

S. De Grazia

Dipartimento di Igiene e Microbiologia, Università di Palermo, Palermo, Italy

\section{G. Pezzotti}

Istituto Zooprofilattico Sperimentale di Umbria/Marche, Perugina, Italy

\section{A. Lavazza}

Istituto Zooprofilattico Sperimentale di Lombardia/Emilia Romagna, Brescia, Italy and phylogenetic analysis of the calicivirus-positive samples characterized the majority as genogroup III (GGIII) sapoviruses. Unclassified caliciviruses, distantly related to the representatives of the other sapovirus genogroups, were identified in five herds, while one outbreak was associated with a porcine sapovirus related genetically to human GGII and GGIV sapovirus strains. By converse, norovirus strains were not detected. Altogether, these data suggest the epidemiological relevance of porcine enteric caliciviruses and suggest a role in the etiology of piglets diarrhoea.

Keywords Porcine calicivirus - Sapovirus - Enteritis

\section{Introduction}

The family Caliciviridae includes small non-enveloped viruses of approximately $35 \mathrm{~nm}$ in diameter with singlestranded, positive-polarity RNA genomes of 7.4-8.3 kb [1]. The family includes the Vesivirus, Lagovirus, Norovirus, and Sapovirus genera as well as unassigned viruses [2]. The member viruses of each genus share a common genomic organization and a high degree of sequence similarity $[2,3]$.

Molecular techniques have revolutionized the diagnostics of noroviruses (NoVs) and sapoviruses ( $\mathrm{SaVs}$ ) and led to the recognition that enteric caliciviruses are responsible for the majority of non-bacterial gastroenteritis in humans of all age groups and important pathogens in childhood diarrhoea [3-7]. In addition, analysis of the polymerase and capsid genes of NoVs and SaVs has provided evidence for a huge genetic diversity. Due to the extent of genetic variation, to the existence of numerous recombinant strains and to the lack of consistency and agreement among enteric calicivirus research groups, classification under the genogroup level is controversial. NoVs have been classified into 
five separate genogroups (GGI to GGV) and at least 29 genotypes [8]. Genetic analysis of $\mathrm{SaV}$ has identified five genogroups (GGI to GGV) and at least 13 genetic clusters or genotypes $[3,9]$.

The ecology of caliciviruses is largely unexplored but there are several pieces of evidences that suggest the possibilities of transmission of viruses between different animal species. Vesivirus infection has been documented in humans in more occasions and in a multiplicity of clinical outcomes (encephalitis, hepatitis, myocarditis, pneumonia, spontaneous abortion and dermatitis) [10-12]. Also, antibodies to marine and terrestrial vesiviruses and vesivirus genome sequences have been detected in blood donors in the United States [13]. Animal enteric caliciviruses genetically related to human NoVs and $\mathrm{SaVs}$ have been detected in pigs and cows [2,14-21]. Antibodies to human NoV have been detected in pigs and antibodies to bovine NoV have been detected in humans [22, 23]. Porcine NoVs have been detected sporadically and are classified into three distinct genotypes of the genogroup GGII (GGII.11, GGII.18, and GGII.19), which are genetically related to the 16 human genotypes of GGII NoVs [8, 19, 24]. The porcine SaVs strains Cowden and LL14/02/US, representatives of $\mathrm{SaV}$ genogroup GGIII, have been detected from diarrheic piglets and have been shown to induce enteric diseases and lesions in experimentally infected pigs [20, 25-28]. Porcine enteric caliciviruses (PECs), distantly related to the porcine $\mathrm{SaV}$ prototype Cowden, have been also described, that are likely members of novel SaV genogroups, GGVI (prototype strain JJ681/ 00/US) and GGVII (prototype strains K7/JP and K10/JP) $[20,29]$. Genetic recombination has been described frequently between genetically related caliciviruses, within the same genogroup or genotype [30,31] but it may also have occurred between genetically unrelated strains [32]. As the molecular epidemiology of animal caliciviruses is largely unexplored, the understanding of the global ecology of NoVs and SaVs is limited and the role of animals as reservoirs of infections is unclear. Accordingly, it remains to be established whether some animal $\mathrm{NoV}$ or $\mathrm{SaV}$ have emerged over time in humans by direct interspecies transmission or by exchange of genetic material via intraor inter-genogroup recombination with related humans caliciviruses.

In this study, we investigated the role of PECs in the aetiology of weaning or post-weaning diarrhea of piglets by reverse transcription-PCR (RT-PCR) using broadly reactive primers targeted to the conserved motifs "DYSKWDST" and "YGDD" of the RNA-dependent RNA polymerase (RdRp) of caliciviruses [7]. To draw a more complete picture of PEC molecular epidemiology in pigs, sequence analysis of the fragment of the RdRp polymerase was also accomplished.

\section{Materials and methods}

Stool samples and viruses

Fecal samples were collected from piglets with either weaning or post-weaning diarrhea at 118 swine farms between 2003 and 2006 in Northern and Central Italy. The pigs were aged between 1 and 3 months. A total of 103 samples (collection A) were collected at the diagnostic unit of the Istituto Zooprofilattico of Umbria and Marche, Perugia, Italy, from 12 outbreaks of enteritis in piglets (5-12 samples per outbreak) in the regions of Umbria and Marche. The samples were screened for the presence of PEC and of group A rotavirus RNA. To investigate the presence of PEC RNA, broadly reactive primers, that are able to amplify members of the Vesivirus, Lagovirus, Norovirus, and Sapovirus genera, were used [7, 33].

The presence of PEC was also assessed in samples obtained from the diagnostic section of the Istituto Zooprofilattico of Lombardia and Emilia Romagna, Brescia, Italy, that were collected during a passive surveillance study for rotavirus in the regions of Lombardia and Emilia Romagna. The samples were screened by immuno-electron microscopy observation and were selected after the presence of rotavirus-like particles (87 samples, collection B) or after the presence of enterovirus-like particles (19 samples, collection C). Each of the 106 samples was representative of a distinct outbreak and it was obtained by either sampling a single animal or by pooling 3-5 faecal samples.

Diagnosis of infection by group A and C rotavirus was also accomplished. Detection of group A rotavirus was carried out using a primer pair targeted to the 4th genome segment that codes for the VP4 capsid protein [34]. Diagnosis of group $\mathrm{C}$ rotavirus infection was accomplished on collection A (103 samples) with a primer pair designed to amplify the $3^{\prime}$ end of the viral VP6 gene [35].

\section{RNA extraction and RT-PCR}

The RNA was extracted from $200 \mu \mathrm{l}$ of $10 \%$ (wt/vol) fecal suspensions in phosphate buffered saline (PBS), using guanidinium isothiocyanate/silica method described by Boom et al. [36]. RNA was eluted in $50 \mu 1 \mathrm{H}_{2} \mathrm{O}$ DEPC with RNasin $(0.2 \mu \mathrm{g} / \mu \mathrm{l}$, Promega) and used in RT-PCR.

RT and PCR were performed in a one-step procedure using Superscript III One step (Invitrogen, UK). A mix of primers p289, p290, p289hi p290hijk was used as previously reported [7, 33] to amplify amplicons of about $329 \mathrm{bp}$. RT-PCR products were analyzed on agarose gel electrophoresis and stained with ethidium bromide (Table 1). The RT-PCR product bands were visualized by using UV light. 
Table 1 List of the oligonucleotides used in this study. Sequence and position are reported as indicated in the original studies

${ }^{\text {a }}$ Gentsch et al. [34]

b Jiang et al. [33]

c Zints et al. [7]

d Sanchez-Fauquier et al. [35]

\begin{tabular}{|c|c|c|c|}
\hline Target & $\begin{array}{l}\text { Primers } \\
\text { (sense) }\end{array}$ & Sequence $\left(5^{\prime}-3^{\prime}\right)$ & Position \\
\hline GARVs & $\begin{array}{l}\operatorname{Con}^{\mathrm{a}}(+) \\
\operatorname{Con}^{\mathrm{a}}(-)\end{array}$ & $\begin{array}{l}\text { tgg ctt } \mathrm{cgc} \text { cat ttt ata gac a } \\
\text { att tcg gac cat tta taa cc }\end{array}$ & $\begin{array}{l}\text { nt } 11-32,4 \text { th dsRNA segment (VP4) } \\
\text { nt } 868-887,4 \text { th dsRNA segment (VP4) }\end{array}$ \\
\hline Calicivirus & $\begin{array}{l}\mathrm{p} 290^{\mathrm{b}}(+) \\
\mathrm{p} 289^{\mathrm{b}}(-) \\
\mathrm{P} 28 \mathrm{~h}^{\mathrm{c}}(-) \\
\mathrm{P} 28 \mathrm{i}^{\mathrm{c}}(-) \\
\mathrm{P}^{\mathrm{c}} 90 \mathrm{~h}^{\mathrm{c}}(+) \\
\mathrm{P} 290 \mathrm{i}^{\mathrm{c}}(+) \\
\mathrm{P} 290 \mathrm{j}^{\mathrm{c}}(+) \\
\mathrm{P} 290 \mathrm{k}^{\mathrm{c}}(+)\end{array}$ & $\begin{array}{l}\text { gat tac tcc aag tgg gac tcc ac } \\
\text { tga caa tgt aat cat cac cat a } \\
\text { tga cga ttt cat cat cac cat a } \\
\text { tga cga ttt cat cat ccc cgt a } \\
\text { gat tac tcc agg tgg gac tcc ac } \\
\text { gat tac tcc agg tgg gac tca ac } \\
\text { gat tac tcc acc tgg gat tca ac } \\
\text { gat tac tcc acc tgg gat tcc ac }\end{array}$ & $\begin{array}{l}\text { nt } 4568-4590, \text { RdRp region of the polymerase } \\
\text { nt } 4886-4865, \text { RdRp region of the polymerase }\end{array}$ \\
\hline GCRVs & $\begin{array}{l}\mathrm{F}(\mathrm{BMJ} 45)^{\mathrm{d}}(+) \\
\mathrm{R}(\mathrm{BMJ} 44)^{\mathrm{d}}(-)\end{array}$ & $\begin{array}{l}\text { agc cac ata gtt cac att tc } \\
\text { agt ccg ttc tat gtg att } c\end{array}$ & $\begin{array}{l}\text { nt } 1334-1353,5 \text { th } d s R N a \text { segment (VP6) } \\
\text { nt } 1032-1014,5^{\text {th }} \text { dsRNa segment (VP6) }\end{array}$ \\
\hline
\end{tabular}

Sequencing analysis

The RT-PCR amplicons of 55 samples were selected because the intensity of the bands by agarose gel visualization and ethidium staining was discrete and the DNA was purified by using a QIAquick gel extraction kit (QIAGEN, Inc.). The amplicons were sequenced directly using primers p290 and p289. DNA sequencing was done by using BigDye Terminator Cycle chemistry and 3730 DNA Analyzer (Applied Biosystems, Foster, CA).

Phylogenetic analysis

Sequence editing and multiple alignments were performed with Bioedit software package vers. 2.1 [37]. Basic Local Alignment Search Tool (BLAST; http://www.ncbi.nlm.nih. gov) and FASTA (http://www.ebi.ac.uk/fasta33) with default values was used to find homologous hits. Phylogenetic analysis (neighbor-joining) with bootstrap $(1,000$ replicates) was conducted by using the MEGA software package v3.0 [38].

\section{Results}

Detection of PEC and group A and C rotaviruses

Out of 103 samples, 30 (collection A) were found positive to calicivirus RNA, whereas 73 and 32 were positive to group A and group C rotavirus RNA, respectively. Mixed infections with two pathogens were identified in a total of 39 samples (PEC + rotavirus $\mathrm{A}, n=10$; $\mathrm{PEC}+$ rotavirus $\mathrm{C}, n=2$; rotavirus $\mathrm{A}+$ rotavirus $\mathrm{C}, n=13$;), and 14 samples were positive for all three viruses. PEC was the single identified enteric virus in four samples $(3.8 \%)$. In a total of 21 diarrheic samples $(20.3 \%)$ no viral pathogen was identified (Table 2).

Therefore, PEC were detected in 30/103 samples $(29.1 \%)$ and in 8 out of 12 herds $(66.6 \%)$. High prevalence
Table 2 Relative distribution of three enteric viruses (PEC, GRAV and GCRV) in fecal specimens of diarrheic pigs

a The selection criteria in this sample collection has been the presence of rotavirus-like particles in the feces

b These samples were determined positive for rotavirus by electron microscopy, but molecular methods failed to recognize GCRVs or GARVs

\begin{tabular}{lllll}
\hline & Identified viruses & \multicolumn{2}{l}{ Sample collection } & \\
\cline { 3 - 5 } & & $\mathrm{A}(n=103)$ & $\mathrm{B}(n=87)$ & $\mathrm{C}(n=19)$ \\
\hline Single infections & PEC & 4 & $1^{*}$ & 2 \\
& GCRV & 3 & 2 & 1 \\
\multirow{2}{*}{ Mixed } & GARV & 36 & 36 & 4 \\
infections & PEC + GCRV & 2 & $4^{\mathrm{a}}$ & 2 \\
& PEC + GARV & 10 & $18^{\mathrm{a}}$ & 3 \\
& PEC + GCRV + GARV & 14 & $6^{\mathrm{a}}$ & 2 \\
& GCRV + GARV & 13 & 10 & 2 \\
& Total GCRV & 32 & 22 & 7 \\
Negative & Total GARV & 73 & 70 & 11 \\
& Total PEC & 30 & $29^{\mathrm{a}}$ & 9 \\
\hline
\end{tabular}


values of PEC infection was found only in two herds. In one herd PEC prevalence was $90 \%$ but group $\mathrm{C}$ rotaviruses were also detected in $70 \%$ of the animals. In another herd the prevalence of PECs was $100 \%$ but group A and C rotaviruses were also detected in $100 \%$ of the samples.

A total of 87-rotavirus positive samples (collection B), each representative of a distinct outbreak (farm), were assayed by RT-PCR for calicivirus and PECs were detected in 29 samples (33.3\%). Eighteen samples contained mixed infection $\mathrm{PEC}+$ rotavirus $\mathrm{A}, 4$ samples were mixed infections $\mathrm{PEC}+$ rotavirus $\mathrm{C}, 6$ samples were positive to all three viruses and 1 sample was a single PEC infection.

In addition, calicivirus RNA was searched for in 19 samples from collection $\mathrm{C}$, selected after the presence of small rounded viral particles by electron microscopy observation (group C), and PEC RNA was found in nine samples (47.3\%). Three such PEC-positive samples were also positive to rotavirus $A, 2$ were positive to rotavirus $C$ and 2 were positive to both rotavirus $A$ and $C$ by RT-PCR.

In summary, a total of 209 samples from 118 herds were screened and PECs were detected in 68 samples $(32.5 \%)$ and in 46 farms $(38.9 \%)$.

Sequence and phylogenetic analysis of the RdRp of PEC strains

A total of 38 out of 55 samples was sequenced successfully, obtaining sequences suitable for comparison and analysis. The PEC-specific sequences were 270-290 nucleotide (nt) long and encoded 90-96 amino acids (aa) of the RdRp region. Six samples were clones of the same sequivars within the same outbreaks/farms, and they were not included in the comparison. Therefore, 31 distinct PEC strains were obtained, each representative of a distinct farm outbreak. After we performed BLAST and FASTA searches, all the sequences contained the GLPSG conserved motif of the calicivirus RdRp and 25 strains were identified as GGIII SaVs. Six strains (42/05-6c, 43/06-18p3, 43/06-64 and 43/04-65, 200/05-10 and 200/05-32) were not classifiable as GGIII $\mathrm{SaV}$ and they were regarded as 'atypical' $\mathrm{SaVs}$. None of the PEC strain was characterized as NoV.

Neighbor-joining and UPGMA phylogenetic analysis was performed based on the 270-nt RdRp sequences of PECs. Representatives of the other genera of caliciviruses, Lagovirus/RHDV/GH/1988/GE, Vesivirus/FCV/F9/1958/US, Norovirus/Norwalk/68/US, and Bo/Nebraska/80/US (the proposed new genus Becovirus), and the canine calicivirus Ca48/1990/JP, tentatively classified in the genus Vesivirus [39] were included in the analysis (Fig. 1). We also included in our analysis the porcine $\mathrm{SaV}$ prototypes Cowden and LL14 and Cowden-like PEC strains (GGIII) available in the databases, such as QW152 and MM280. The PEC strains JJ681 and K7/JP and K10/JP, that are likely the prototypes of new $\mathrm{SaV}$ genogroups (GGVI and GGVII) [20, 29], respectively, were also included, along with other atypical PEC strains listed in GenBank. In addition, a variety of human GGI, GGII, GGIV, GGV, SaV and mink $\mathrm{SaV}$ strains were included.

The overall intra-genogroup amino acid (aa) identity of the $\mathrm{RdRp}$ region of $\mathrm{SaVs}$ is $80-100 \%$ and this parameter was applied to distinguish tentatively the relationships among the various strains. The $\mathrm{SaV}$ strains analyzed in this study were clustered in at least five different genetic clusters. Most PEC strains were GGIII SaV, Cowden-like. In the tree, GGIII SaVs formed a well-defined cluster (bootstrap value 98\%). Within this cluster, three clear lineages were defined. The major lineage (I) included all the Cowden-like SaVs (bootstrap value 98\%), from United States, Korea, Venezuela, the Netherlands, and Italy while the minor lineages II included Korean and Hungarian strains (bootstrap value 97\%) and the minor lineage III included the American strains MM280 and QW152 and two additional $\mathrm{SaVs}$ from the Netherlands (bootstrap value 99\%). The overall aa identity within GGIII $\mathrm{SaV}$ was roughly $>80 \%$. The majority of the SaVs obtained in this study segregated within the major lineage I, suggesting a tight homology with the prototype strains Cowden and LL14. The identity within lineage I GGIII SaV was 94$100 \%$. However, identity of lineage I to lineage II SaVs was $90-94 \%$ and to lineage III SaVs was 81-85\%. Our data suggest that within GGIII $\mathrm{SaV}$ there are at least three distinct polymerase (pol) types. The three lineages were inferred on the basis of a short fragment of the polymerase and therefore they are pol types. Due to the possibility of recombination, the pol types do not necessarily reflect different capsid genotypes. Indeed, sequence analysis of the capsid gene of strain MM280 (pol type III) has revealed 91-93\% aa identity to strain Cowden and LL14 (pol type I), suggesting that this strain is a recombinant, possessing the same capsid genotype as strains Cowden and LL14 but a different pol type [20].

The SaV strain 43/06-18p3 was grouped with strains QW19 (United States), SWECII/VA103 and SWECII/ VA14 (the Netherlands). Identity to the SaV strains within this group was 97-98\% aa while identity to the genetically most closely related SaVs, the human Lyon/97 and Mc10 (GGII) and Hou7-1181 and SW278/2004/SE (GGIV) was $65-67 \%$. Identity to other SaVs was $<50 \%$ aa.

Several additional groups were resolved in the tree that included atypical $\mathrm{SaVs}$ from this and other studies and that were highly supported statistically. Strains 200/05-32, 43/ 06-64, and 43/06-65 were grouped along with strains SWECIII/VA24a, K8, K15, and K24 (bootstrap values $99 \%$ ). All the strains within this group shared more than $87 \%$ aa identity, while identity to strains SWEC/VA112 and $42 / 05-6 \mathrm{C}$ was $<76 \%$ aa. 
Fig. 1 Neighbor-joining phylogenetic tree based on the partial RdRp region (270 nt) of SaVs and RHDV, NB, Norwalk, FCV, CaCV strains. The newly identified atypical PECs are in boldface

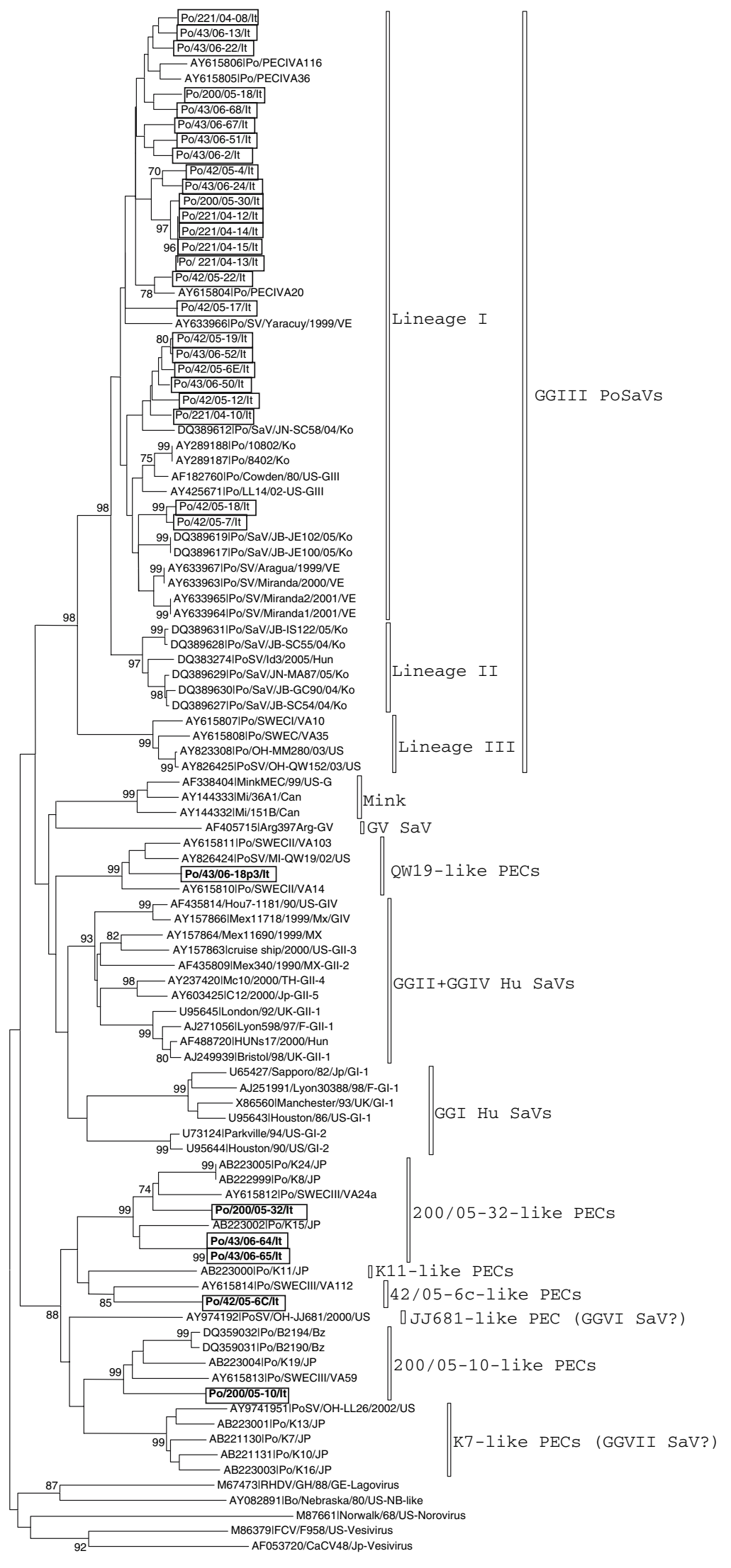

10 
Strain 42/05-6C clustered along with strain SWEC/ VA112 (97\% aa) (bootstrap values $85 \%$ ) while it was more distantly related to strains within the 200/05-32-like group (72-75\% aa).

The SaV strain 200/05-10 was clustered along with the porcine strains K19/JP and SWECIII/VA59 (bootstrap value $99 \%$ ). Identity within this group was $>84 \%$ aa, while lower aa identities were found to strains of the new $\mathrm{SaV}$ group K7-like (64-67\%, bootstrap value 99\%) and to strain JJ681 (63\%). Identity to strains within the 200/05-32-like group was $<50 \%$ aa.

To evaluate the consistency in estimating the genetic similarity of strains and genogroups, a histogram showing the frequency distribution of the pairwise distances was used to determine the differentiability of the intragenogoup, intergenogroup and intergenera distances. To resolve the genetic relationships among the atypical SaVs, members of the five well-established Sapovirus genogroups (GGI to GGV) and members of other calicivirus genera, the alignment of the 270-nt fragment of the RdRp was used to generate a plot of the nucleotide distance (p-distance) (Fig. 2a). In this analysis, the intragenogroup distance (within each genogroup) was $0-31 \%$, while the intergenogroup distance (between members of different genogroups) ranged between $32 \%$ and $49 \%$. The intergenera distance (between members of different calicivirus genera) was equal or higher than $50 \%$. By plotting into this model the genetic distance of the genetic groups resolved by phylogenetic analysis, it appeared that QW19-like strains are a novel Sapovirus genogroup (Fig. 2b), while 200/05-32-like SaVs (Fig. 2c), 42/05-6C-like SaVs (Fig. 2d) and 200/05-10-like SaVs (Fig. 2e) are distantly related, genetically, to the various Sapovirus genogroups and their taxonomical collocation is unclear.

\section{Discussion}

In this survey evidence was collected for a wide distribution of $\mathrm{SaVs}$ in swine herds where enteritis outbreaks were described. SaV infection was demonstrated in 30/103 samples (29.1\%) and 8 out of 12 herds (66.6\%), alone or in mixed infections with group $\mathrm{A}$ and $\mathrm{C}$ rotaviruses (collection A). The presence of SaVs was also investigated in samples collected from enteritis outbreaks, selected by electron microscopy after the presence of rotavirus-like particle
Fig. 2 Histograms showing the frequency distribution of pairwise p-distances between 81 nucleotide sequences, including Sapovirus members (GGI to GGV), representatives of the other calicivirus genera (Vesivirus, Norovirus,

Lagovirus) and atypical PECs

(a). Arrows indicate the range of the nt distance comparison within genogroups, between genogroups and between genera. Histograms plotting the genetic relatedness of QW19like PECs (b), 200/05-32-like PECs (c), 42/05-6C-like PECs (d) and of 200/05-10-like PECSs. Distances were calculated by the p-distance algorithm of correction using a $270 \mathrm{nt}$ fragment of the $\mathrm{RdRp}$
Intragenogroup distance range (d.r.) Intergenogroup d.r. Intergenera d.r.

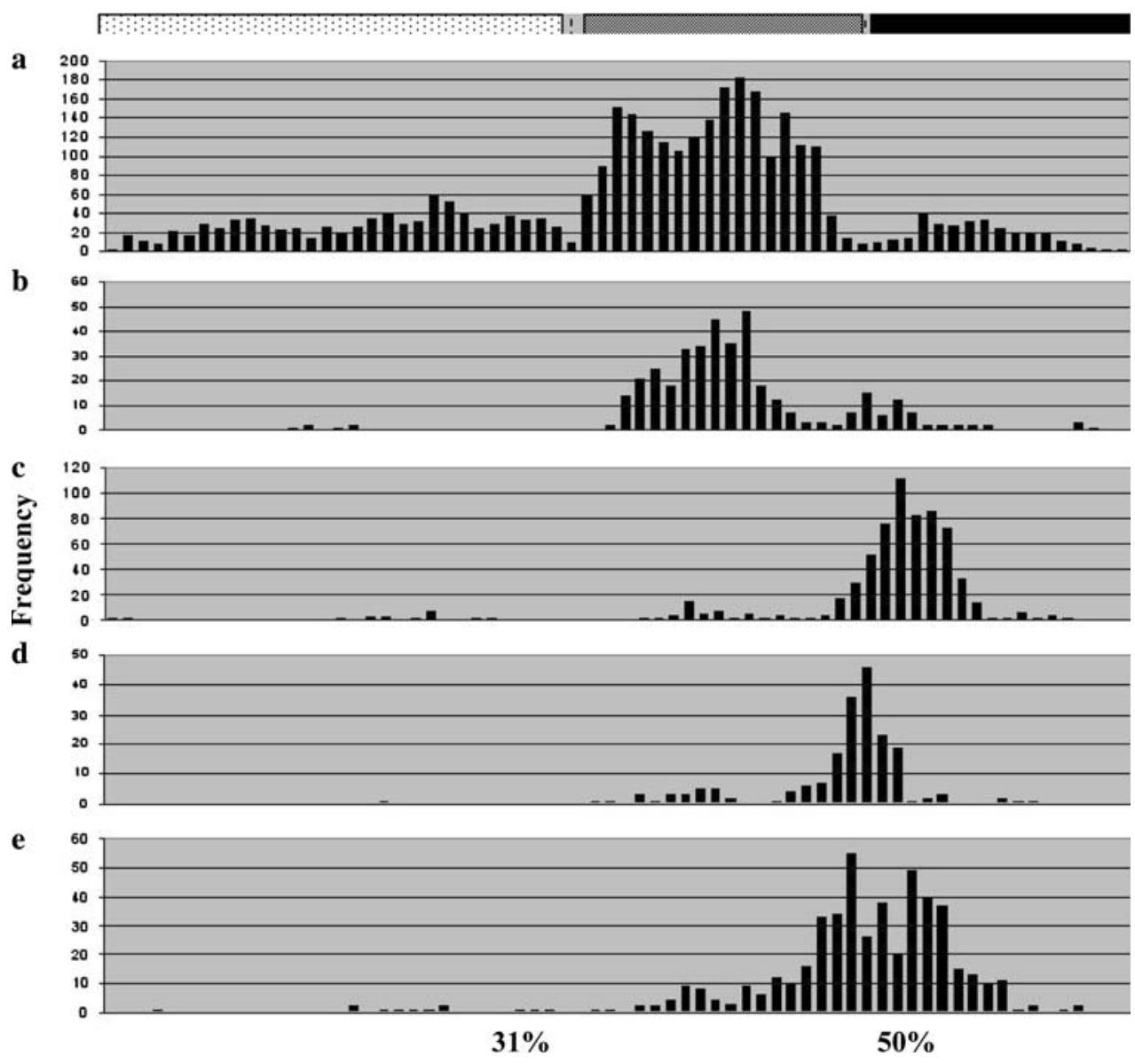


(collection B) or small-rounded viral particles (collection C). In these samples collections, porcine $\mathrm{SaVs}$ were detected in 38/106 outbreaks. Overall, porcine SaVs were detected in 46 out of 118 distinct outbreaks. This is the first study to provide the results of a large-scale survey and to demonstrate that $\mathrm{SaVs}$ are widespread in porcine herds with weaning or post-weaning enteritis. However, due to the selection criteria of our samples collections, a true prevalence data could be determined only for sample set A. By converse, the possibility to investigate the presence of $\mathrm{SaVs}$ in different outbreaks allowed us to gather important information on the genetic heterogeneity of $\mathrm{SaVs}$ in pigs.

Early in the study of porcine enteric caliciviruses, investigations based on collection of rectal swabs from slaughtered animal or from healthy animals revealed very low rates of PEC (SaVs and NoVs) infections. PECs were detected only in 4 of 1,017 normal slaughtered pigs in Japan, in 2 of 100 pooled pig fecal samples of 3- to 9month-old fattening pigs in the Netherlands and in 14 out of 377 healthy adult and finisher pigs in the United States [18-20, 40]. These findings, while documenting the existence of porcine $\mathrm{NoV}$ and $\mathrm{SaV}$ and their genetic relationships with human enteric caliciviruses, raised a number of questions, with particular regard to their pathogenic attitude and impact in pigs. Subsequent studies carried out on both healthy and symptomatic pigs at different ages have contributed to draw a more complete picture. Analysis of 102 faecal samples of nursing and weaning pigs with enteritis from 31 farms in Korea has revealed a low prevalence $(8.8 \%)$ and all the samples were characterized as GGIII SaVs [41]. Analysis of 203 fecal samples collected from seven farms in a 10-years time span in Venezuela has revealed a high frequency of detection of GGIII SaVs (Cowden-like) in pigs between 3 and 4 weeks of age (39\%), with almost equal frequencies of detection in healthy and diarrhoeic animals [42]. The existence of agebased restriction mechanisms has been clearly evidenced in a more comprehensive study in United States [43]. Analysis of 610 samples collected from healthy animals and of 11 samples collected from symptomatic animals from seven farms and one slaughterhouse in Ohio, Michigan and Northern Carolina, has revealed an high overall prevalence $(62 \%)$ of $\mathrm{SaVs}$ in all age groups, ranging from $21 \%$ in nursing pigs (1-3 weeks old) to $83 \%$ in post-weaning pigs (3-10 weeks old). These findings indicate that porcine $\mathrm{SaVs}$ are common enteric viruses in piglets but do not prove definitively their aetiological role in piglets enteritis, as SaVs may be identified both in healthy and in symptomatic animals. However, the pathogenic potential of porcine $\mathrm{SaVs}$ has been unequivocally demonstrated in experimental infections. Oral inoculation of 4-day-old gnotobiotic pigs inoculated with wild-type strain Cowden, GGIII $\mathrm{SaV}$, resulted in profuse diarrhoea, anorexia, and intestinal lesions [26]. In addition to PEC, other enteric viruses, such as group $\mathrm{A}$ and $\mathrm{C}$ rotaviruses, are commonly detected in young pigs. In our study, we could find mixed infections in nearly $37.9 \%$ of the animals (39/103) in collection A. Also, 27/29 and 7/9 PEC-positive samples from collection $\mathrm{B}$ and $\mathrm{C}$ were mixed infections with either rotavirus $\mathrm{A}$ or $\mathrm{C}$ or both. It may hypothesized that mixed infections may trigger synergetic effects by the various enteric pathogens and this may occur more frequently in intensive herds, where animals are kept overcrowded, and at the post-weaning phase, when pigs from the various piglets are mixed and are under social and environmental stress.

In our analysis, porcine NoV strains were not detected. This is in agreement with previous investigation targeting asymptomatic and older animals and revealing sporadic patterns of detection [18, 19, 40, 43]. Likewise, NoVs could be detected only from healthy finisher pigs (with prevalences varying from $0 \%$ to $40 \%$ ) or from slaughtered animals (prevalence 14\%) but not from nursing, postweaning pigs or from adult sows ( $>1$ year) [43] suggesting age-restricted patterns of susceptibility to infection by NoV. In our study the age of the animals ranged from 1 to 3 months. Accordingly, it may be hypothesized that NoV are not implied in the aetiology of diarrhoea in weaning and post-weaning piglets or that they play only a minor epidemiological role.

Molecular analysis revealed that all the PECs analyzed in this study belong to the Sapovirus genus. The majority of the SaVs were classified as GGIII. Sequence and phylogenetic analysis allowed us to identify unusual $\mathrm{SaVs}$, that accounted for $19.3 \%(6 / 31)$ of the confirmed calicivirus outbreaks. Unusual SaVs were clustered in at least four novel potential genetic groups, distantly related to GGIII $\mathrm{SaV}$ (Cowden-like) and to JJ681-like and K7-like SaVs. Due to the recombination phenomenon, a consistent and reliable classification of $\mathrm{SaVs}$ is necessarily based on the complete capsid gene sequences, and $\mathrm{SaV}$ classification based on different regions, such as the complete and partial $\mathrm{RdRp}$ remains to be assessed [3, 7]. However, as the $\mathrm{RdRp}$ region contains highly conserved motives, it represents the best target for the construction of broadly reactive primers that may be applied for molecular detection of a variety of $\mathrm{SaV}$ genogroups and calicivirus genera. Accordingly, investigation of the genetic relationships among the various PECs in the RdRp fragment is important to provide a tool for comparison of large data sets and, with some exceptions, may be regarded as a good proxy for strains characterization. In this and other studies, classification based on the short segment (95 aa) of the RdRp region could be used to characterize strains into genogroups and to identify novel potential calicivirus groups. The overall intragenogroup amino acid identity of the $\mathrm{RdRp}$ region of 
$\mathrm{SaVs}$ ranges from $80 \%$ to $100 \%$. Applying this cut-off value, we could approximate characterize the atypical PEC strains into seven novel potential genetic groups, as resolved in our phylogenetic reconstruction. The six atypical PEC strains segregated in four such genetic groups. Strains 200/05-32, 43/06-64, and 43/06-65 were grouped along with strains SWECIII/VA24a (the Netherlands) and $\mathrm{K} 8$, K15, and K24 (Japan). All the strains within this group shared more than $87 \%$ aa identity but displayed $<76 \%$ identity to the other SaVs.

Strain 42/05-6C clustered along with strain SWEC/ VA112 (97.7\% aa) while it was more distantly related to strains within the 200/05-32-like cluster ( $<76 \%$ aa). The $\mathrm{SaV}$ strain 200/05-10 was clustered along with the porcine strains K19/JP and SWECIII/VA59. All the strains in this group shared $>84.8 \%$ aa identity and displayed $<67.0 \%$ aa identity to $\mathrm{K} 7$-like $\mathrm{SaVs}$ and $63.2 \%$ aa identity to strain JJ681 (proposed as a GGVI SaV). All these genetic groups (200/05-32, 42/05-6C, and 200/05-10-like) may represent candidate new $\mathrm{SaV}$ genogroups. Analysis of the genetic distance in the short fragment of the RdRp region (Fig. 2c-e) does not support unambiguous classification into the Sapovirus genus. Accordingly, determining the sequence of the full-length $3^{\prime}$ end and the genome organization is necessary to understand more precisely their taxonomical collocation. Also, experimental infection of gnotobiotic pigs is required to evaluate the pathogenic potential in pigs.

The PEC strain 43/06-18p3 was grouped with strains QW19 (United States), SWECII/VA103 and SWECII/ VA14 (the Netherlands). These strains shared $>97 \%$ aa identity and represent the cluster, genetically most closely related to members of the main human $\mathrm{SaV}$ genogroups (up to $67 \%$ aa identity). Analysis of the genetic distance (Fig. 2b) supports the inclusion of QW19-like PECs into the Sapovirus genus but also suggests that QW19-like PEC represent a candidate novel $\mathrm{SaV}$ genogroup. The detection of QW19-like strains in pigs from different geographic areas (Europe and United States) suggests that, although rare, the natural host of Q19-like viruses is the swine. The strain 43/06-18p3 was detected in a swine farm in Brescia in 2005, in a geographic area where there is a high density of swine farms. Whether QW19-like strains may circulate in humans, such as in workers of the swine industry is unknown. Molecular epidemiology of humans enteric calicivirus infections will be extremely important to acquire information on the zoonotic potential of these porcine SAVs.

In summary, we demonstrated the epidemiological relevance of PECs in enteritis outbreaks in piglets and we obtained information on PEC genetic heterogeneity. One PEC strain was found to resemble QW19-like porcine $\mathrm{SaVs}$ that are genetically similar to human GGII and GGIV
SaVs, suggesting the possibility of direct zoonotic transmissions or of meeting points between pigs and humans during $\mathrm{SaV}$ evolution.

Acknowledgments We thank Narcisi Donato for his technical collaboration. The work was supported by grants from Progetto di Ricerca finanziato con fondi di Ateneo (ex quota 60\%) "Studio dei calicivirus animali ed implicazioni zoonosiche".

\section{References}

1. A.Z. Kapikian, M.K. Estes, R.M. Chanock, in Fields Virology, 3rd edn. ed. by B.N. Fields, D.M. Knipe, P.M. Howley (Raven Press, New York, 1996), pp. 783-810

2. S.L. Oliver, E. Asobayire, A.M. Dastjerdi, J.C. Bridger, Virology. 350, 240 (2006)

3. T. Farkas, W.M. Zhong, Y. Jing, P.W. Huang, S.M. Espinosa, N. Martinez, A.L. Morrow, G.M. Ruiz-Palacios, L.K. Pickering, X. Jiang, Arch. Virol. 149, 1309 (2004)

4. L.H. Blanton, S.M. Adams, R.S. Beard, G. Wei, S.N. Bulens, M.A. Widdowson, R.I. Glass, S.S. Monroe, J. Infect. Dis. 193, 413 (2006)

5. C.I. Gallimore, M. Iturriza-Gomara, D. Lewis, D. Cubitt, H. Cotterill, J.J. Gray, J. Med. Virol. 78, 673 (2006)

6. B. Lopman, H. Vennema, E. Kohli, P. Pothier, A. Sanchez, A. Negredo, J. Buesa, E. Schreier, M. Reacher, D. Brown, J. Gray, M. Iturriza, C. Gallimore, B. Bottiger, K.O. Hedlund, M. Torven, C.H. von Bonsdorff, L. Maunula, M. Poljsak-Prijatelj, J. Zimsek, G. Reuter, G .Szucs, B. Melegh, L. Svennson, Y. van van Duijnhoven, M. Koopmans, Lancet 363, 682 (2004)

7. C. Zintz, K. Bok, E. Parada, M. Barnes-Eley, T. Berke, M.A. Staat, P. Azimi, X. Jiang, D.O. Matson, Infect. Genet. Evol. 5, 281(2005)

8. D.P. Zheng, T. Ando, R.L. Fankhauser, R.S. Beard, R.I. Glass, S.S. Monroe, Virology 346, 312 (2006)

9. G.S. Hansman, T. Oka, K. Katayama, N. Takeda, Rev. Med. Virol. 17, 133 (2007)

10. A.W. Smith, E.S. Berry, D.E. Skilling, J.E. Barlough, S.E. Poet, T. Berke, J. Mead, D.O. Matson, Clin. Infect. Dis. 26, 434 (1998)

11. A.W. Smith, D.O. Matson, D.A. Stein, D.E. Skilling, A.D. Kroeker, T. Berke, P.L. Iversen, Curr. Opin. Mol. Ther. 4, 177 (2002)

12. A.W. Smith, D.E. Skilling, N. Cherry, J.H. Mead, D.O. Matson, Emerg. Infect. Dis. 4, 13 (1998)

13. A.W. Smith, P.L. Iversen, D.E. Skilling, D.A. Stein, K. Bok, D.O. Matson, J. Med. Virol. 78, 693 (2006)

14. C.A. Batten, I.N. Clarke, S.L. Kempster, S.L. Oliver, J.C. Bridger, P.R. Lambden, Virology 356, 179 (2006)

15. Y. Deng, C.A. Batten, B.L. Liu, P.R. Lambden, M. Elschner, H. Gunther, P. Otto, P. Schnurch, W. Eichhorn, W. Herbst, I.N. Clarke, J. Clin. Microbiol. 41, 2300 (2003)

16. S.L. Oliver, C.A. Batten, Y. Deng, M. Elschner, P. Otto, A. Charpilienne, I.N. Clarke, J.C. Bridger, P.R. Lambden, J. Clin. Microbiol. 44, 992 (2006)

17. S.L. Oliver, A.M. Dastjerdi, S. Wong, L. El-Attar, C. Gallimore, D.W. Brown, J. Green, J.C. Bridger, J. Virol. 77, 2789 (2003)

18. W.H. van der Poel, J. Vinje, R. van Der Heide, M.I. Herrera, A. Vivo, M.P. Koopmans, Emerg. Infect. Dis. 6, 36 (2000)

19. Q.H. Wang, M.G. Han, S. Cheetham, M. Souza, J.A. Funk, L.J. Saif, Emerg. Infect. Dis. 11, 1874 (2005)

20. Q.H. Wang, M.G. Han, J.A. Funk, G. Bowman, D.A. Janies, L.J. Saif, J. Clin. Microbiol. 43, 5963 (2005) 
21. K. Mattison, A. Shukla, A. Cook, F. Pollari, R. Friendship, D. Kelton, S. Bidawid, J.M. Farber, Emerg. Infect. Dis. 13, 1184 (2007)

22. T. Farkas, S. Nakajima, M. Sugieda, X. Deng, W. Zhong, X. Jiang, J. Clin. Microbiol. 43, 657 (2005)

23. M.A. Widdowson, B. Rockx, R. Schepp, W.H. van der Poel, J. Vinje, Y.T. van Duynhoven, M.P. Koopmans, J. Med. Virol. 76, 119 (2005)

24. M. Sugieda, S. Nakajima, Virus. Res. 87, 165 (2002)

25. W.T. Flynn, L.J. Saif, J. Clin. Microbiol. 26, 206 (1988)

26. W.T. Flynn, L.J. Saif, P.D. Moorhead, Am. J. Vet. Res. 49, 819 (1988)

27. M. Guo, K.O. Chang, M.E. Hardy, Q. Zhang, A.V. Parwani, L.J. Saif, J. Virol. 73, 9625 (1999)

28. M. Guo, J. Hayes, K.O. Cho, A.V. Parwani, L.M. Lucas, L.J. Saif, J. Virol. 75, 9239 (2001)

29. M. Guo, J. Hayes, K.O. Cho, A.V. Parwani, L.M. Lucas, L.J. Saif, J. Virol. 75, 9239 (2001)

30. G. Reuter, K. Krisztalovics, H. Vennema, M. Koopmans, G. Szucs, J. Med. Virol. 76, 598 (2005)

31. G. Reuter, H. Vennema, M. Koopmans, G. Szucs, J. Clin. Virol. 35, 84 (2006)

32. G.S. Hansman, N. Takeda, T. Oka, M. Oseto, K.O. Hedlund, K. Katayama, Emerg. Infect. Dis. 11, 1916 (2005)
33. X. Jiang, P.W. Huang, W.M. Zhong, T. Farkas, D.W. Cubitt, D.O. Matson, J. Virol. Methods 83, 145 (1999)

34. J.R. Gentsch, R.I. Glass, P. Woods, V. Gouvea, M. Gorziglia, J. Flores, B.K. Das, M.K. Bhan, J. Clin. Microbiol. 30, 1365 (1992)

35. A. Sanchez-Fauquier, E. Roman, J. Colomina, I. Wilhelmi, R.I. Glass, B. Jiang, Arch. Virol. 148, 399 (2003)

36. R. Boom, C.J. Sol, M.M. Salimans, C.L. Jansen, P.M. Wertheimvan Dillen, J. van der Noordaa, J. Clin. Microbiol. 28, 495 (1990)

37. R. Boom, C.J. Sol, M.M. Salimans, C.L. Jansen, P.M. Wertheimvan Dillen, J. van der Noordaa, J. Clin. Microbiol. 28, 495 (1990)

38. S. Kumar, K. Tamura, M. Nei, Brief Bioinform 5, 150 (2004)

39. Y. Matsuura, Y. Tohya, K. Nakamura, M. Shimojima, F. Roerink, M. Mochizuki, K. Takase, H. Akashi, T. Sugimura, Virus Genes 25, 67 (2002)

40. M. Sugieda, H. Nagaoka, Y. Kakishima, T. Ohshita, S. Nakamura, S. Nakajima, Arch. Virol. 143, 1215 (1998)

41. H.J. Kim, H.S. Cho, K.O. Cho, N.Y. Park, J. Vet. Med. B. Infect. Dis. Vet. Public Health. 53, 155 (2006)

42. M.A. Martinez, A.C. Alcala, G. Carrubo, L. Botero, F. Liprandi, J.E. Ludert, Vet. Microbiol. 116, 77 (2006)

43. Q.H. Wang, M. Souza, J.A. Funk, W. Zhang, L.J. Saif, J. Clin. Microbiol. 44, 2057 (2006) 